\title{
EXPERIMENTAL STUDY OF PARAMETRIC INSTABILITIES DRIVEN BY FINITE-EXTENT LOWER-HYBRID WAVES*
}

\author{
J. E. Stevens and W. D. GeTtY \\ Electron Physics Laboratory, Department of Electrical and Computer Engineering, The University of \\ Michigan, Ann Arbor, MI 48109, U.S.A.
}

(Received 19 August 1980; and in revised form 18 December 1980)

\section{INTRODUCTION}

THE LOWER-HYBRD wave is an attractive candidate for the supplemental heating of Tokamak plasmas because of its engineering advantages in a reactor environment. It is estimated that $50 \mathrm{MW}$ of supplementary r.f. heating power will be required to ignite a DT fusion reactor. At this power level the linear theory for lower-hybrid waves may be invalid, and one is forced to consider possible non-linear effects such as parametric instabilities. This paper presents experimental results on the parametric decay of the lower-hybrid 'pump' wave into another lower-hybrid wave and a non-resonant 'quasi-mode', that is, a wave which is not a normal mode of the plasma. PORKOLAB (1974) has shown that this decay process is dominant in the regime $\omega<2-3 \omega_{L H}$ which may constitute the main bulk of the plasma, where $\omega_{L H}$ is the local lower-hybrid frequency. The quasi-mode process may be an important heating mechanism due to strong damping of the short wavelength decay waves. The ATC Tokamak lower-hybrid heating experiment described by PORKOLAB et al. (1977), gave evidence that non-resonant parametric decay was present and involved in heating, and the characteristic spectrum of non-resonant decay provided a diagnostic means of assessing lower-hybrid wave penetration into the hot Tokamak plasma.

With this motivation, a study of this decay process in a linear plasma device was undertaken for the particular purpose of observing non-resonant parametric decay in the presence of lower hybrid wave resonance conss. These are set up by an electrostatic wave coupler in the manner described by BELLAN and PORKOLAB (1976) and COLESTOCK and GETTY (1976). The presence of a resonance cone structure in the pump wave rather than a homogeneous pump field structure like that used by CHANG and PORKOLAB (1974) allows one to observe saturation of the decay wave by convection out of the local pump region. PORKOLAB (1978) predicted that convection of the decay wave is the most likely saturation mechanism for this instability.

Previous experimental work in linear plasma devices on quasi-mode parametric instabilities includes the dispersion relation studies of CHAVG and PORKOLAB (1974) in a uniform pump field, and observations of efficient plasma heating related to the quasi-mode decay by Bonizzoni et al. (1977). STEVENS and GETTY (1980) have reported observations of quasi-mode and ion-acoustic wave decay spectra and wavelength measurements. Linear cold plasma devices can operate in the same relative parameter range as fusion devices in lower-hybrid heating

\footnotetext{
* This work was supported by the National Science Foundation.
} 
experiments insofar as frequency ratios are concerned (i.e. $\omega_{0} / \omega_{L H}<3, \omega_{L H} / \omega_{\mathrm{pi}} \simeq$ 1 , and $\omega_{p e} / \omega_{c e}<1$ ), and resonance cone effects are expected as in fusion experiments. Therefore, the results obtained from linear devices should aid in interpreting fusion heating experiments. The relative r.f. power levels are comparable for the central regions (i.e. where $\omega / \omega_{L H}<3$ ) of linear machines and Tokamaks, where $\varepsilon_{0} E_{0}{ }^{2} / n k T_{e} \simeq 0.001-0.002$ in current heating experiments. However, near the source it is likely that this parameter will be approximately unity in Tokamaks.

The new results presented here are measurements of the quasi-mode decay process in a linear laboratory plasma for $\omega / \omega_{\mathrm{LH}} \simeq 2.5$ along with corresponding numerical results based on Porkolab's theory. Specifically, measurements and calculations of the dispersion characteristics, threshold, instability growth above noise, and saturation levels were made for the quasi-mode decay process. Heating measurements were not made. Section 2 of this paper presents the relevant theory and Section 3 gives numerical results based on this theory. The experimental setup is described in Section 4 and the experimental results are presented and compared with theory in Section 5. The results are summarized in Section 6.

\section{THEORY}

The process considered in this paper is a wave-wave-particle process involving parametric decay of a finite-extent lower-hybrid wave into another lower-hybrid wave and a non-resonant quasi-mode. PORKOLAB (1977) has given an approximate solution to the dispersion equation for this process. Assuming that the lowersideband decay wave is a normal mode satisfying the lower-hybrid wave dispersion equation

$$
\omega_{2}^{2}=\omega_{L H}^{2}\left(1+\frac{k_{H 2}^{2}}{k_{2}^{2}} \frac{m_{i}}{m_{e}}\right)
$$

the growth rate is given approximately by

$$
\gamma=-\Gamma_{2}-\frac{\mu^{2}}{4\left|\left(\partial \varepsilon_{R}-\partial \omega_{2}\right)\right|} \operatorname{Re}\left[\frac{i \chi_{e}\left(\omega_{1}\right) \chi_{i}\left(\omega_{1}\right)}{\varepsilon\left(\omega_{1}\right)}\right]
$$

where $\Gamma_{2}$ is the collisional and Landau damping rate of the sideband and the other symbols have the following definitions:

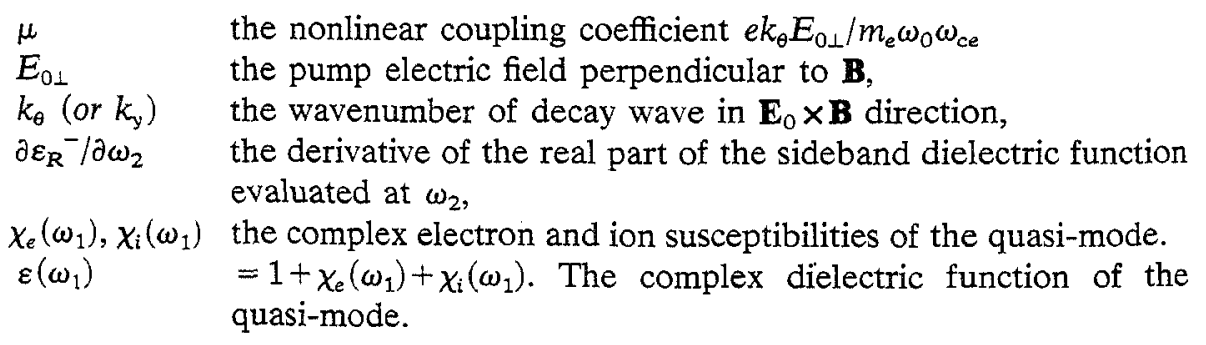

The frequency and wavenumber matching conditions also apply:

$$
\omega_{0}=\omega_{1}+\omega_{2}
$$

and

$$
\mathbf{k}_{0}=\mathbf{k}_{1}+\mathbf{k}_{2}
$$




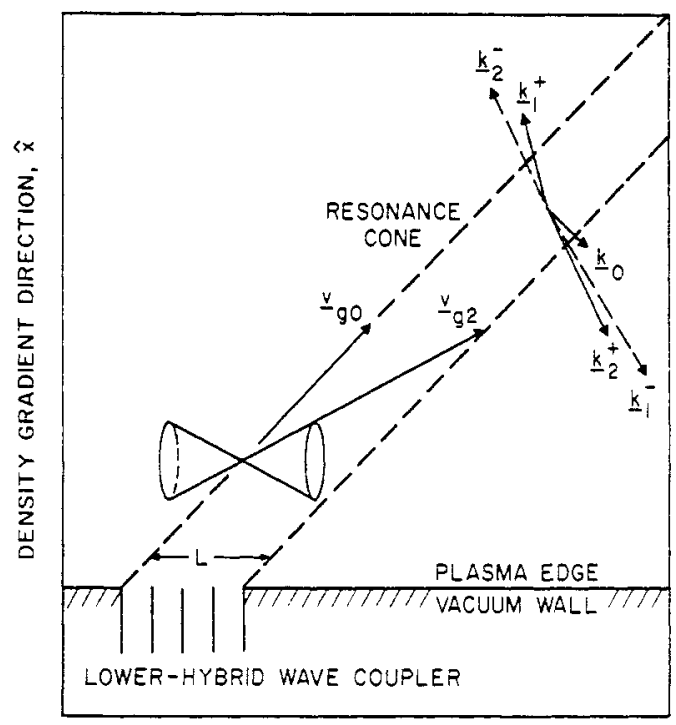

MAGNETIC FIELD DIRECTION, $\hat{z}$

FIG. 1.-Idealized diagram of lower-hybrid wave experiment showing the directions of the group velocities and wavenumbers for the pump and the decay waves. The decay wavenumbers labeled with a "-" are for the case when the direction of the sideband group velocity $\mathbf{v}_{\mathrm{g} 2}$ is reversed.

where the subscripts $0,1,2$ denote pump, quasi-mode and sideband, respectively. The decay process described by (2) is unstable if $\gamma>0$, i.e., it grows as $\exp (\gamma t)$. This occurs for strong pump fields since $\mu$ is proportional to $E_{0}$.

To analyze saturation of the unstable sideband fluctuation by convection out of the pump resonance cone one must consider the resonance cone geometry and group velocities of the waves as illustrated in Fig. 1. The pump wave is launched by a phased array structure of waveguides for a Tokamak or an electrostatic coupler for a small laboratory plasma. The pump wave is confined to a spatially localized resonance cone region as shown by BELLAN and PORKOLAB (1976). The directions of the pump wave group velocity $\left(\mathbf{v}_{\mathrm{g} 0}\right)$ and sideband group velocity $\left(\mathbf{v}_{\mathrm{g} 2}\right)$ are shown along with the wavenumbers for the pump $\left(\mathbf{k}_{0}=k_{x 0} \hat{x}+k_{z 0} \hat{z}\right)$, sideband $\left(\mathbf{k}_{2}=k_{\mathrm{x} 2} \hat{x}+k_{\mathrm{y} 2} \hat{y}+k_{z 2} \hat{z}\right)$ and quasi-mode $\left(\mathbf{k}_{1}=k_{\mathrm{x} 1} \hat{x}+k_{\mathrm{y} 1} \hat{y}+k_{z 1} \hat{z}\right)$. The decay wavenumbers labeled with $a(+)$ are for the case where $\mathbf{v}_{\mathrm{g} 2}$ has the direction shown, while those labeled with a (-) are for the case where $\mathbf{v}_{82}$ has the direction opposite to that shown. Since the sideband is a lower-hybrid wave it must also propagate in resonance cones. The double cone shown in Fig. 1 indicates the possible directions of propagation for a sideband fluctuation originating at the vertex. One line on the surface of each cone will have the maximum growth for the unstable sideband wave.

Quantitatively, the number of $e$-foldings of an unstable sideband wave as it traverses the resonance cone is given by

$$
\Gamma=\int_{\text {pump }} \frac{\gamma(z)}{v_{\mathrm{s} z 2}} \mathrm{~d} z
$$

where $\gamma(z)$ is the temporal growth rate defined in (2), and $v_{s z 2}$ is the group 
velocity of the sideband in the $\hat{z}$ direction. The integral is taken over a $\hat{z}$ distance for which the sideband remains inside the localized pump wave. The growth rate equation for an infinite uniform plasma with a uniform pump is assumed to hold locally for the region inside the pump wave, and the growth rate is assumed to be zero outside the pump region. The final amplitude of an unstable fluctuation is given by

$$
\phi_{2 \max }=\phi_{2 \text { noise }} \cdot \mathrm{e}^{\Gamma}
$$

where the unstable wave is assumed to start from a background noise level. VILLALON and BERS (1980) give an approximate evaluation of (5) as

$$
\Gamma \cong \frac{\gamma L}{v_{g z 2}\left(1-\frac{v_{g z 0}}{v_{g \times 0}} \frac{v_{g \times 2}}{v_{g z 2}}\right)} .
$$

This expression is maximized as a function of $k_{x 2} / k_{\perp 2}$, giving

$$
\frac{k_{x 2}}{k_{\perp 2}}=\frac{k_{\mid 0} k_{\perp 2}}{k_{\perp 0} k_{\mid 2}}-\left[\left(\frac{k_{\| 0} k_{\perp 2}}{k_{\perp 0} k_{\mid 2}}\right)^{2}-1\right]^{1 / 2} \text {. }
$$

The maximum amplification is a trade-off between the direction of propagation which allows the maximum temporal growth rate $\gamma$ (large $k_{y 2}$ ) and the direction which allows the sideband to remain inside the pump resonance cone the longest (large $k_{x 2}$ ).

It was assumed in deriving (8) that the group velocity of the sideband $\left(v_{\mathrm{gz} 2}\right)$ was in the same direction as the pump wave group velocity $\left(v_{\mathrm{gz} z}\right)$. It is equally possible for the sideband to propagate in the opposite direction and have the same growth rate. It is found numerically that inclusion of a finite $\mathbf{k}_{0}$ will result in different dispersion properties for the two cases, i.e. for waves with $v_{\mathrm{gz} 2}$ having the same or opposite sign as $v_{\mathrm{gz} 0}$.

\section{NUMERICAL RESULTS}

The non-linear dispersion equation (2) for non-resonant parametric decay of a lower-hybrid wave is solved numerically in this section, using the experimental parameters given in Section 4. The general solution to (2) can be written symbolically as

$$
\omega_{1}+i \gamma=F\left(\mathbf{k}_{\perp 2}, k_{\| 2}, \omega_{2}, \mathbf{k}_{\perp 0}, k_{\| 0}, \omega_{0}, \mathbf{E}_{0}, n, \mathbf{B}, \mathcal{T}_{e}, T_{i}, \nu_{e}, \nu_{i}\right)
$$

where $F$ means function of, $\mathbf{k}_{\perp 0}=k_{x 0} \hat{x}, \mathbf{E}_{0}=E_{0} \hat{x}, \mathbf{B}=B \hat{z}, \nu_{e}$ is the electron collision frequency, $\nu_{i}$ is the ion collision frequency, and the other terms have been defined previously. The solution of the exact dispersion equation would involve extensive root searching in the complex plane. This has been done by PORKOLAB (1977), and no attempt will be made to repeat this calculation. The approach taken here is to find $\gamma$ from (2), which assumes the sideband is a weakly damped normal mode. This simplifies the calculation by allowing for the direct solution of the sideband frequency $\omega_{2}$ using (1). The sideband damping $\Gamma_{2}$ is a 
combination of collisionless and collisional terms given by

$$
\begin{aligned}
\Gamma_{2}=\left[\left.\frac{\partial \varepsilon_{R}}{\partial \omega}\right|_{\omega,}\right]^{-1} & \varepsilon_{I}\left(\omega_{2}\right)=\frac{\omega_{2}}{2\left(1+\frac{\omega_{p e}{ }^{2}}{\Omega_{e}{ }^{2}}\right)} \\
& \left\{\frac{\sqrt{ } \pi \xi_{2}}{k_{2}{ }^{2} \lambda_{D}{ }^{2}} \exp \left(-\xi_{2}{ }^{2}\right)+\frac{\nu_{c e}}{\omega_{2}}\left(\frac{\omega_{p e}{ }^{2} k_{\| 2}{ }^{2}}{\omega_{2}{ }^{2} k_{2}{ }^{2}}+\frac{\omega_{p e}{ }^{2} k_{\perp 2}{ }^{2}}{\Omega_{e}{ }^{2} k_{2}{ }^{2}}\right)+\nu_{c i} \frac{\omega_{p i}{ }^{2}}{\omega_{2}{ }^{3}}\right\}
\end{aligned}
$$

where $\xi_{2}=\omega_{2} / k_{\| 2} v_{t e}$ and $\lambda_{\mathrm{D}}{ }^{2}=k_{\mathrm{B}} T_{e} / 4 \pi n e^{2}$. The frequency matching condition (3) is used to solve for $\omega_{1}$ directly and $\gamma$ is then calculated from (2).

The calculation proceeds by fixing all but the first three parameters on the right hand side of (9). Then $\omega_{1} / \omega_{0}$ from (3), $\gamma / \omega_{0}$ and $\Gamma$ are plotted on a contour plot in the $k_{12} \lambda_{D}-k_{\mid 2} \lambda_{D}$ plane. The sideband wavenumber $\mathbf{k}_{2}$ is plotted rather than the quasi-mode wavenumber $\mathbf{k}_{1}$, because the sideband wavenumber is an experimentally measured quantity.

There is an additional degree of freedom in $\mathbf{k}_{\perp 2}$ because it can have both an $\hat{x}$ and a $\hat{y}$ component. Equation (8) was used to specify the ratio $k_{x 2} / k_{12}$ which gives the largest convective growth.

Still another degree of freedom exists for the calculation if the dipole approximation is relaxed, i.e. if a finite $\mathbf{k}_{0}$ for the pump wave is allowed. The effect of $\mathbf{k}_{0}$ is included in (2) by replacing $\mathbf{k}_{2}$ with $\mathbf{k}_{0}-\mathbf{k}_{1}$ in the definition of electron and ion susceptibilities and by multiplying $\mu$ by the factor $\left(\omega_{0} / \omega_{2}\right)\left(k_{1} / k_{2}\right)$, as discussed by PORKOLAB (1977). The case where the sideband axial group velocity $v_{g z 2}$ is in the same direction as the pump wave axial group velocity $v_{g z 0}$ will be referred to as the 'co-directional' case, and the opposite situation will be referred to as the 'contra-directional' case. It can be shown by considering the parallel wavenumber matching conditions for the two cases that if $k_{\| 1}$ is assumed to be approximately fixed by the condition $\omega_{1} / k_{\| 1}=v_{t e} / \sqrt{2}$, then $k_{\mid 2}$ for the co-directional case will be larger than $k_{\| 2}$ for the contra-directional case. This was observed experimentally as well as in the numerical solutions presented in this Section.

Although a finite $\mathbf{k}_{0}$ can be included in the dispersion equation, it cannot be made larger than either $\left|\mathbf{k}_{1}\right|$ or $\left|\mathbf{k}_{2}\right|$. Decay waves with wavelengths larger than the pump resonance cone width would no longer be fully contained inside the pump field resonance cone. Since the lower-hybrid pump wave resonance cone width is approximately only one pump wavelength wide in the experiment, the uniform pump-field approximation is valid only for $\left|\mathbf{k}_{1}\right|,\left|\mathbf{k}_{2}\right|>\left|\mathbf{k}_{0}\right|$. Only those regions of $\mathbf{k}_{2}$ space which satisfy this condition are plotted in the numerical results.

A case based on the experimental parameters of Section 4 is now considered. A pump field of $\mathbf{E}_{\text {ox }}=20 \mathrm{~V} \mathrm{~cm}^{-1}, \lambda_{00}=30 \mathrm{~cm}$ and $f_{0}=35 \mathrm{MHz}$ is present in a uniform helium plasma of density $n=2.7 \times 10^{10} \mathrm{~cm}^{-3}, T_{e}=5.5 \mathrm{eV}, T_{i}=0.2 \mathrm{eV}$ and $B_{0}=850 \mathrm{G}$. The electric field is approximately twice the threshold value. The value of $\omega_{0} / \omega_{L H}$ is 2.4 .

Figure 2 is a plot of the normalized growth rate $\gamma / \omega_{0}$ vs $k_{\perp_{2}} \lambda_{D}$ and $k_{\| 2} \lambda_{D}$ for all unstable roots $(\gamma>0)$. The sideband and pump are assumed to be codirectional. The components of $\mathbf{k}_{2}$ are normalized to the Debye length. The location of the normalized value of the perpendicular pump wavenumber is 


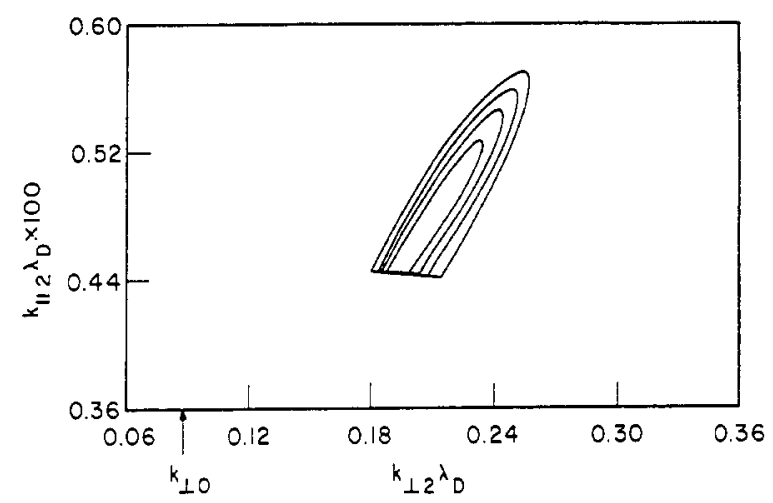

FIG. 2.-Normalized growth rate $y / \omega_{0}$ as a function of $k_{\lambda_{2}} \lambda_{D}$ and $k_{\| 2} \lambda_{D}$ for the co-directional case. Each contour represents an increment of 0.004 . The maximum value is $\gamma_{\mathrm{MAX}} / \omega_{0} \cong 0.014$. The plasma parameters are given in the text.

indicated with an arrow on the plot. The normalized value of the pump parallel wavenumber is $k_{\mid 0} \lambda_{D}=0.0022$, but $\left|\mathbf{k}_{1}\right|>\left|\mathbf{k}_{0}\right|$ only for $k_{\| 2} \lambda_{D}>0.0044$. Each contour in Fig. 2 represents an increment of $\gamma / \omega_{0}=0.004$. The outer contour $(\gamma=0)$ divides the $k_{\perp 2} \lambda_{D}-k_{\mid 2} \lambda_{D}$ plane into regions of stability and instability. The maximum growth rate indicated in Fig. 2 is $\gamma_{\operatorname{Max}} / \omega_{0} \cong 0.014$.

The real part of the dispersion equation is plotted in Fig. 3. Each contour represents an increment of 0.05 in normalized frequency $\omega_{1} / \omega_{0}$. The outermost contour is determined by $\gamma / \omega_{0}=0$ as in Fig. 2. The leftmost contour (small $k_{\perp}$ side) is at zero frequency while the rightmost contour (large $k_{\perp}$ side) is at $\omega_{1} / \omega_{0} \cong 0.15$.

The spatial amplification factor $\Gamma$ defined by (5) and (7) has contour plots very similar to $\gamma / \omega_{0}$ in Fig. 2. The maximum number of $e$-foldings of the instability is $\Gamma_{\text {MAX }} \cong 0.6$ for the data of Figs. 2 and 3. The amplification is small for this case because $E_{0}$ is only about twice the threshold value.

The unusual feature of the quasi-mode parametric decay instability is the wide range of wavenumber space which is unstable. This is possible because the low frequency quasi-mode does not have to satisfy a unique dispersion relation.

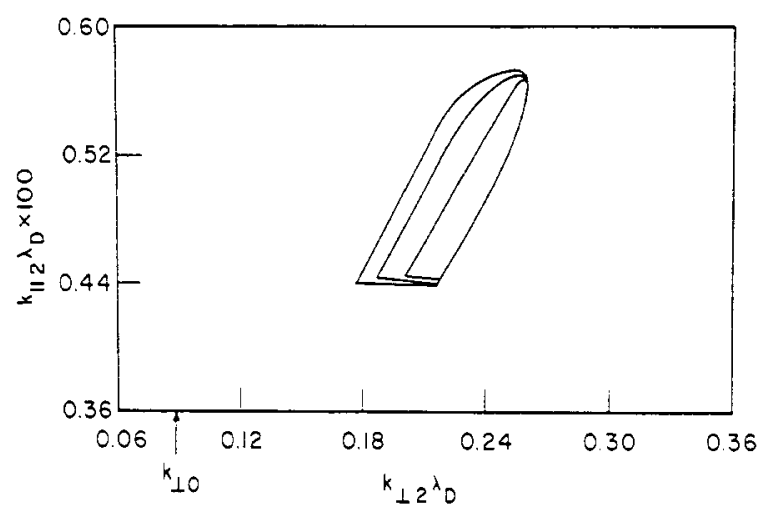

Frg. 3.-Normalized quasi-mode frequency $\omega_{1} / \omega_{0}$ as a function of $k_{12} \lambda_{D}$ and $k_{\| 2} \lambda_{D}$. All parameters are the same as in Fig. 2. Three contours are shown which coalesce along the large $k_{1}$ side. Each contour represents an increment of 0.05 . 
Instead it satisfies the approximate condition $\omega_{1} / k_{\| 1} \cong v_{\text {te }} / \sqrt{2}$, which allows $\omega_{1}$ and $\mathbf{k}_{1}$ to take on a range of values and still satisfy the matching conditions. The unstable range of quasi-mode frequency $\omega_{1}$ is very wide, ranging up to $15 \%$ of $\omega_{0}$. One would expect to see this broad spectrum experimentally on a spectrum analyzer. This would contrast with the resonant type of decay which occurs for only a narrow range of $\omega_{1}$ and $\mathbf{k}_{1}$.

Similar contour plots were calculated for the case of contra-directional group velocities. Figures 4 and 5 show the results for this case, with all other data identical to that of the co-directional case given in Figs. 2 and 3. In this case the allowed ranges of $k_{\perp 2}$ and $k_{\| 2}$ are larger without violating $\left|\mathbf{k}_{1}\right|>\left|\mathbf{k}_{0}\right|$, and the range of unstable wavenumbers is larger. The maximum growth rate from Fig. 4 is $\gamma_{\operatorname{MAX}} / \omega_{0} \cong 0.018$ which is $30 \%$ larger than the co-directional case. The normalized frequency $\omega_{1} / \omega_{0}$ ranges up to 0.30 in Fig. 5 which is also much larger than in the case of a co-directional sideband. The maximum spatial amplification $\Gamma_{\text {MAX }}$ is approximately the same as the co-directional case.

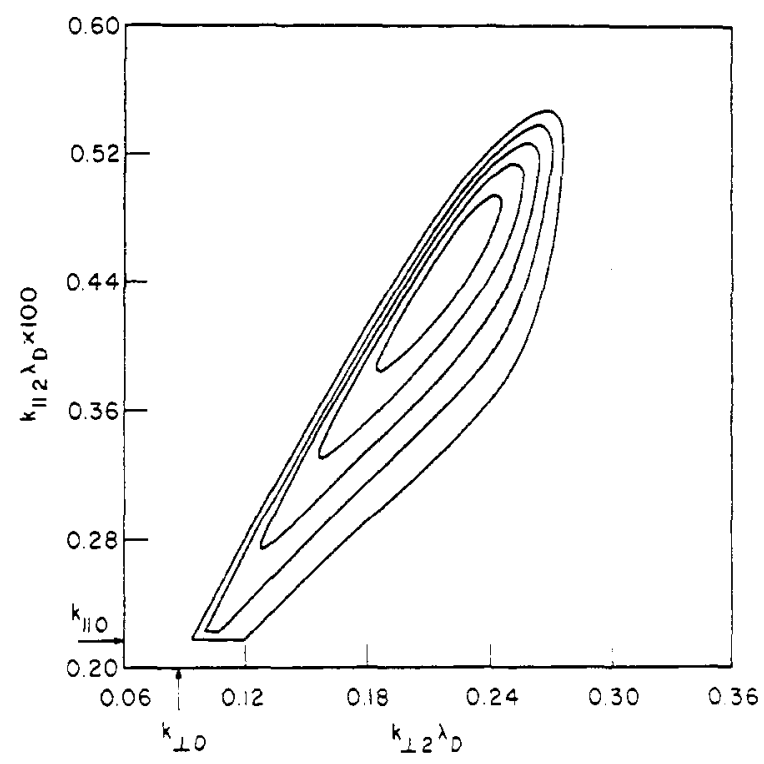

FiG. 4.-Normalized growth rate $\gamma / \omega_{0}$ as a function of $k_{\alpha_{2}} \lambda_{D}$ and $k_{12} \lambda_{D}$ for the contra-directional case. All other parameters are the same as in Fig. 2. Each contour represents an increment of $\gamma / \omega_{0}=0.004$. The maximum value is $\gamma_{\text {MAX }} / \omega_{0} \cong 0.018$.

The parameters $T_{i}, \nu_{e}$ and $\nu_{i}$ affect the parametric instability process in a relatively weak way. The parameters $E_{0}, \omega_{0} / \omega_{L H}, T_{e}$ and $\mathbf{k}_{0}$ have a strong effect on the instability growth rate. Numerical studies were carried out which showed that the maximum growth rate increases linearly with $E_{0}{ }^{2}$ when $E_{0}$ is far above threshold. The maximum growth rate also increases as $\omega_{0} / \omega_{L H}$ approaches 1 , roughly doubling as $\omega_{0} / \omega_{L H}$ is decreased from 2.5 to 1.6. This suggests that the relatively weak unstable waves seen in the experiment $\left(\omega_{0} / \omega_{L H} \equiv 2.5\right)$ would become much stronger as the wave penetrates closer to the lower-hybrid layer $\left(\omega_{0} / \omega_{L H}=1\right)$. Another trend seen in the numerical results is that the maximum growth decreases with increasing temperature. This is partly due to an increase in the Landau damping of the sideband $\left[\Gamma_{2}\right.$ in (2)] which raises the threshold. 


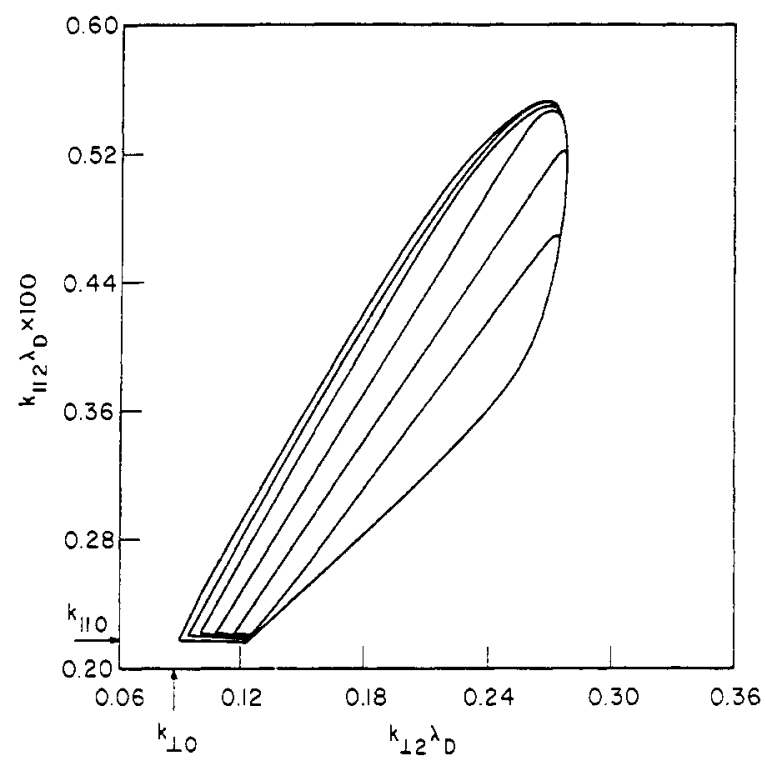

FIG. 5.-Normalized quasi-mode frequency $\omega_{1} / \omega_{0}$ as a function of $k_{{ }_{2}} \lambda_{D}$ and $k_{12} \lambda_{D}$ for the contra-directional case. All other parameters are the same as in Fig. 2. Each contour represents an increment of $\omega_{1} / \omega_{0}=0.05$, with the small $k_{\perp}$ side at zero frequency and the large $k_{\perp}$ side at $\omega_{1} / \omega_{0} \cong 0.30$.

Finally, it was noted that increasing $k_{\| 0}$ had the effect of reducing the region of unstable sideband decay wavenumbers $\left(\mathbf{k}_{2}\right)$. This is because $k_{\| 2}$ is constrained to be larger than $k_{\| 0}$ by the uniform pump approximation and to be smaller than the value $\left(\sim \omega_{2} / 3 v_{r e}\right)$ where Landau damping of the sideband becomes strong (thus raising $\Gamma_{2}$ and the threshold pump field). This effect could reduce the likelihood of exciting this instability as the temperature approaches $10 \mathrm{keV}$.

\section{EXPERIMENT}

The experimental objective was to study parametric quasi-mode excitation in a linear plasma device with a finite-extent resonance cone structure for the pump wave. A diagram of the general experimental arrangement is shown in Fig. 6 . The experiments are performed inside a $10-\mathrm{cm}$ diameter stainless steel vacuum vessel which is $120 \mathrm{~cm}$ in length. Fourteen coils provide an almost uniform magnetic field throughout the experimental region. The plasma source was a helical Lisitano coil excited by microwaves at $2.45 \mathrm{GHz}$. The magnetic field was adjusted to obtain approximate cyclotron resonance in the coil region. The $5-\mathrm{cm}$ inside diameter of the helix determined the nominal diameter of the plasma. Helium was the working gas. Microwave power in the range of 50-100 W was sufficient to produce a plasma with typical average density of 1 to $4 \times 10^{10} \mathrm{~cm}^{-3}$, an average electron temperature from 4 to $8 \mathrm{eV}$ and peak-to-peak density fluctuations of $5 \%$ on the axis. The magnetic field was $850 \mathrm{G}$.

The region of interest $\left(\omega_{0}<3 \omega_{L H}\right)$ for quasi-mode decay waves is $20-40 \mathrm{~cm}$ axially from the four-ring pump-wave coupler because of the slow radial penetration of the resonance cone. A ray-tracing computer program was used to establish possible operating regimes within the spatial region covered by the probes. In 


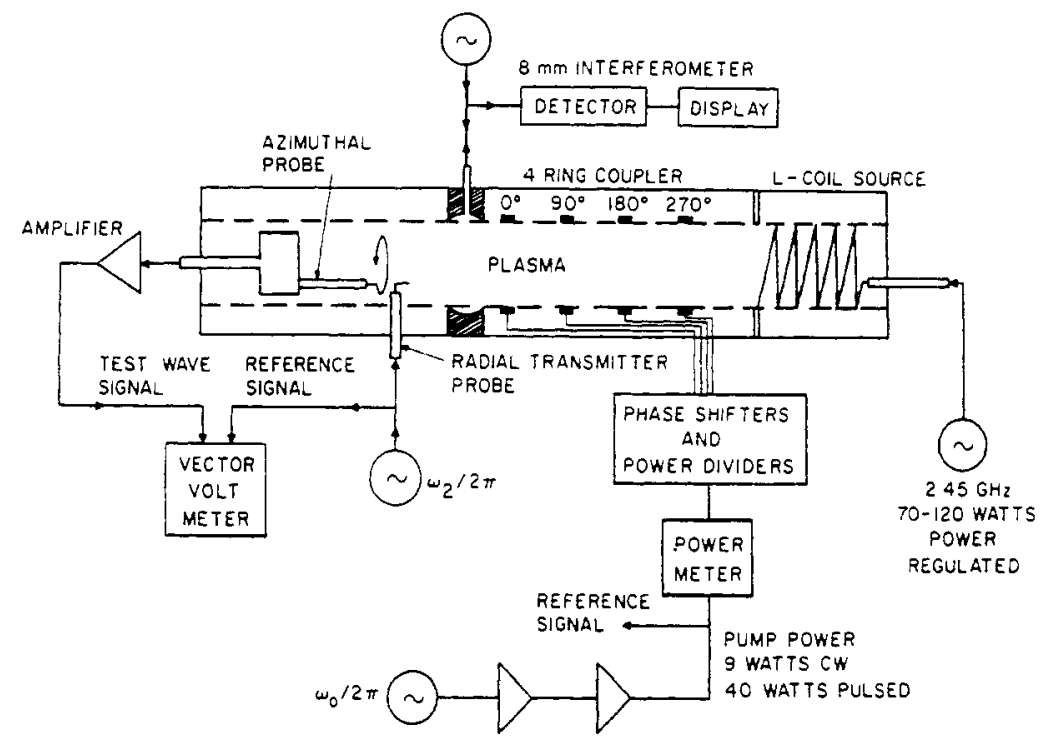

FIG. 6.-Experimental set-up.

order to operate at low values of $\omega_{0} / \omega_{L H}$ and to work away from the noisy plasma edge, one is constrained by the coupler length, probe motion, and plasma length to operate in the range $2<\omega_{0} / \omega_{L H}$ (local) $<3$ and $20 \mathrm{~cm}<z<50 \mathrm{~cm}$, where $z$ is the axial distance from the midplane of the lower-hybrid wave coupler. These conditions imply a frequency range of $30-40 \mathrm{MHz}$ for a typical value of $f_{\mathrm{LH}}(\max ) \cong 15 \mathrm{MHz}$, and radius range for parametric decay of $\Delta r=0.7 \mathrm{~cm}$. When these constraints are combined with those imposed by the dispersion equation for a nonzero growth rate, it is found that quasi-mode decay occurs over a rather narrow range of frequencies and spatial location in this experiment.

The lower-hybrid wave coupler is a set of four copper rings which surround the plasma column and are spaced $7.5 \mathrm{~cm}$ apart. A combination of 180 and 90-degree power splitters were used to drive the rings with successive phase differences of 90 degrees, imposing a parallel wavelength spectrum centered at $30 \mathrm{~cm}$. This choice of wavelength was made to avoid Landau damping of the pump wave and the parametrically excited lower-hybrid-wave sideband. The sideband has a shorter wavelength than the pump and is even more strongly damped. Thus the pump wavelength is located well into the regime of weak Landau damping to reduce damping of the sideband and thereby reduce the instability threshold.

Density measurements are made with Langmuir probes, a Fabry-Perot 35 $\mathrm{GHz}$ interferometer, and by comparison with ray-tracing calculations of the resonance-cone trajectories. Electron temperature is measured by Langmuir probes and by the measured dispersion relation of an electrostatic ion cyclotron test wave. These measurements were reasonably consistent.

Triaxial r.f. probes were used to observe frequency spectra, resonance-cone structure, and pump and sideband wavelengths. These probes were also used as Langmuir probes. They are moveable with motion in the radial, azimuthal, and 
axial directions. An r.f. vector voltmeter is used to detect the phase of a test wave and the resonance cones. A double probe based on a design by BeLLAN and Porkolab (1976) was used to detect the r.f. electric field, and was calibrated in a transmission-line device similar to the design of BROWN and SHEPHERD (1967). It was found that the electric field in the plasma scaled as the square-root of the r.f. power applied to the power divider. The range of power used was $0-9 \mathrm{~W}(\mathrm{CW})$ and $0-50 \mathrm{~W}$ pulsed, but an appreciable fraction of this power was lost in the power divider and phase-shifting circuits.

\section{EXPERIMENTAL RESULTS}

The spectrum shown in Fig. 7 illustrates the quasi-mode decay spectrum observed in this experiment. The low-frequency mode is the quasi-mode and the lower sideband is a lower-hybrid wave (as is the pump). The figure shows the wide spectra of the quasi-mode and sideband which are characteristic of the nonresonant decay process. $\omega_{0} / \omega_{\mathrm{LH}}$ is approximately 2.5 for this case. The frequency of the quasi-mode is centered at $8 \mathrm{MHz}$ which satisfies the condition for nonlinear Landau damping: $\omega_{1} / k_{\| 1} \cong v_{\text {re }} / \sqrt{2}$. Quasi-mode frequencies of up to $30 \%$ of the pump frequency were observed. The frequency matching condition $\omega_{0}=\omega_{1}+\omega_{2}$ is always satisfied.

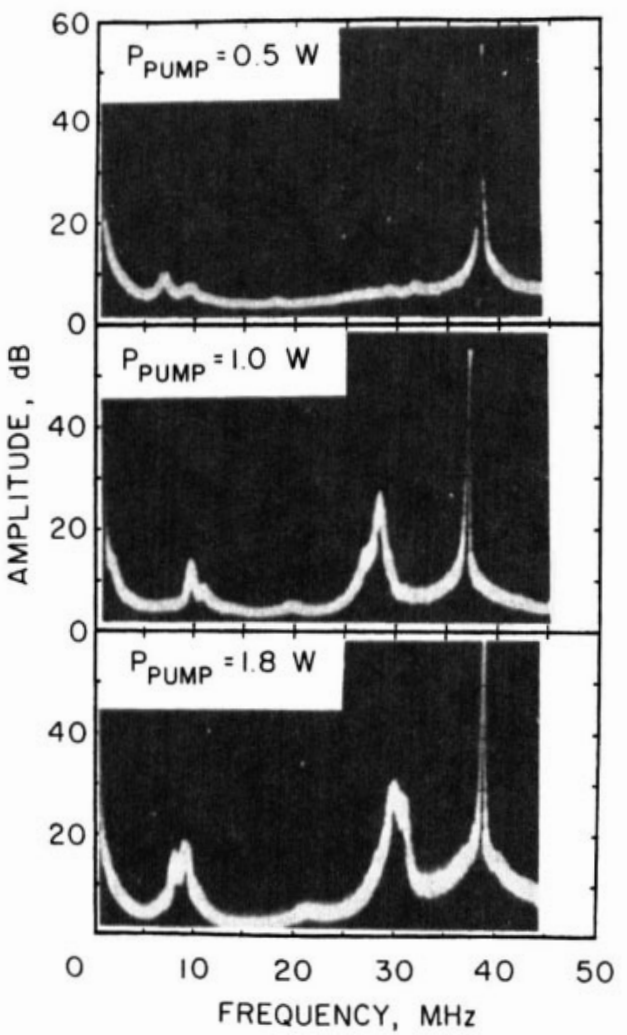

Fio. 7.-Decay wave amplitude versus pump power for powers of (a) $0.5 \mathrm{~W}$, (b) $1.0 \mathrm{~W}$ and (c) $1.8 \mathrm{~W}$. Parameters are $f_{0}=40 \mathrm{MHz}$, peak density $n=2.8 \times 10^{10} \mathrm{~cm}^{-3}$, and helium gas. 
Figure 7 shows the evolution of the decay spectrum for increasing pump power. The pump powers are $0.5,1.0$ and $1.8 \mathrm{~W}$ which correspond to electric field strengths of approximately 4,6 and $8 \mathrm{~V} \mathrm{~cm}^{-1}$ in the resonance-cone region. With a r.f. probe positioned inside the resonance cone and the pump wave on continuously (CW), as the pump power is increased, the quasi-mode and lower sideband first appear together at a power of less than one watt. The quasi-mode frequency is generally between 6 and $10 \mathrm{MHz}$. At this same power level the plasma density begins to change with increasing r.f. power. The density usually decreases with increasing r.f. power, which has the effect of increasing $\omega_{0} / \omega_{L H}$ and quenching the quasi-mode type of decay. This density decrease is counteracted by adjusting the Lisitano-coil plasma source slightly to hold the average density as shown by the interferometer constant. The region of the spectrum between the pump and sideband fills in with increasing pump power. The spectrum shifts over to the ion-acoustic low-frequency decay mode unless the density decrease is compensated for by adjusting the source. Pulsed measurements to be discussed later are more appropriate for studying saturation of decay-wave growth because the problem of density change is reduced.

It is observed that the strongest parametric decay spectra occurred for pump frequencies between 33 and $38 \mathrm{MHz}$ for our plasma. At frequencies above $40 \mathrm{MHz}$ the plasma exhibited the typical spectrum of decay into ion-acoustic waves. Below $33 \mathrm{MHz}$ (down to the $30 \mathrm{MHz}$ limit of the power splitter) the decay-wave amplitude diminishes, even though one would expect even stronger quasi-modes since $\omega_{0} / \omega_{L H}$ is smaller and from (2) one finds that this lowers the threshold. One reason for this behavior may be that there is no more parameter space available to the sideband which is not heavily Landau damped, i.e. $\omega_{2} \approx \omega_{0}, k_{\mid 2}>2 k_{\| 0}$ and $\omega_{0} / k_{\mid 0} v_{\text {te }}$ is near the region where Landau damping becomes strong. Thus since $\omega_{2} / k_{\| 2} v_{t e} \ll \omega_{0} / k_{\| 0} v_{t e}$, the sideband is more strongly damped and the instability is quenched.

Wavelengths of the lower sideband were measured by launching a small amplitude test wave from one probe and receiving that signal on another probe as shown in Fig. 6. One probe is fixed at a position where parametric decay is strongest and the other probe is moved axially, radially, or azimuthally. Phase change is detected by a vector voltmeter and recorded on an $X-Y$ recorder. The wavelength is measured over the range of frequencies for which the lower sideband wave is unstable.

This measurement assumes that the transmitting probe acts as a point source and launches a wide spectrum of wavelengths. Those wavelengths which satisfy the dispersion equation at the particular frequency chosen will propagate to the receiving probe. The test wave frequency was chosen to fall in the center of the broad spectrum of the spontaneously-generated lower sideband, whose frequency spectrum was too wide to permit direct measurement of its wavelength by the lock-in detector method used in the experiment. No test wave signal was observed when the pump was off, showing that, for the output power of the test-wave r.f. signal generator used, the lower-hybrid wave launched by the probe in the absence of a pump wave was too weak to be detected even though it was readily detected when the pump was on. No coherent waves were detected when the excitation of the transmitting probe was turned off, showing that when the 
wavelength signal is observed it is due to the test wave. Coherent waves were easily detected at test-wave frequencies corresponding to the observed peak in the lower sideband spectrum. When the transmitting probe was excited in the frequency range of the quasi-modes, no test wave could be detected.

The wavenumber in the $\theta$ (or $y$ ) direction (which is also the $\mathbf{E}_{0} \times \mathbf{B}_{0}$ direction) determines the non-linear coupling parameter $\mu$. The wavenumber component $k_{\theta 2}=2 \pi / \lambda_{\theta 2}$ is the largest component of $\mathbf{k}_{2}$. Wavelengths in the $\theta$ direction typically ranged from 2 to $5 \mathrm{~mm}$.

The results of these measurements are plotted in Fig. 8 along with theoretical predictions. The experimental parameters are $n(0) \cong 2.3 \times 10^{10} \mathrm{~cm}^{-3}, T_{e} \cong 5.5 \mathrm{eV}$, $f_{0}=35 \mathrm{MHz}, B_{0}=850 \mathrm{G}$, helium gas, pump parallel wavelength of nominally $30 \mathrm{~cm}$, and pump power of approximately $7 \mathrm{~W}$. Two sets of data are presented. The circular symbols and solid error bars represent data taken for the codirectional case, i.e. when the receiving probe is farther from the pump-wave coupler than the transmitting probe. With the roles of the transmitting and receiving probes reversed, the contra-directional case is obtained and is shown by the triangular symbols and dashed line error bars. The error bars represent relative error among the data points.

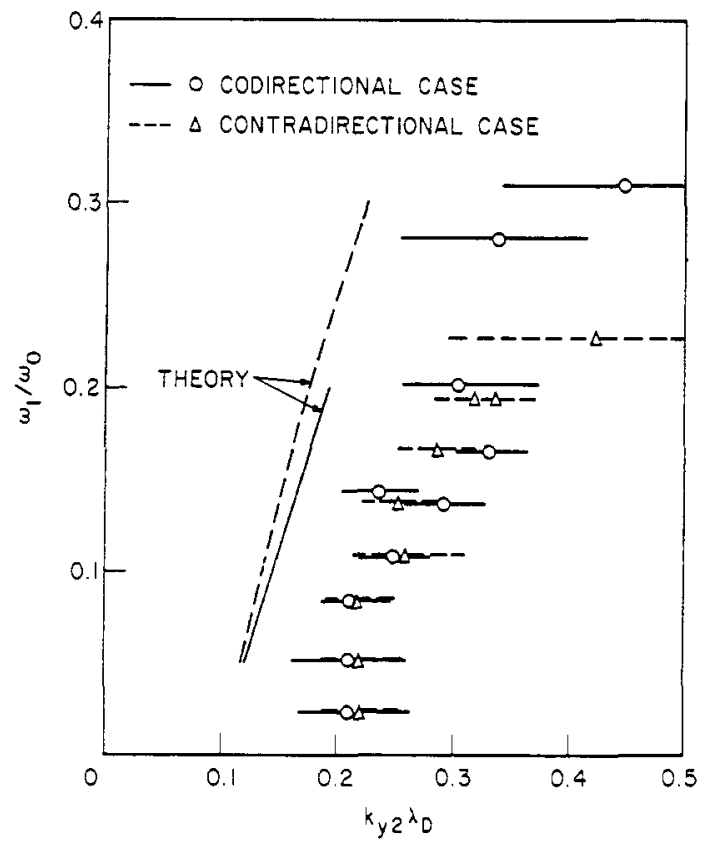

FIG. 8.-Measured sideband wavenumbers $k_{\mathrm{y} 2} \lambda_{\mathrm{D}}$ (or $k_{\theta 2} \lambda_{\mathrm{D}}$ ) vs normalized frequency $\omega_{1} / \omega_{0}$ of the quasi-mode. Within experimental error there is no difference between waves in the $+\theta$ and $-\theta$ directions.

It is seen that shorter wavelengths were measured than were theoretically predicted, but the measured and calculated curves appear to be different by only a multiplicative constant. This systematic error (factor of $\frac{2}{3}$ ) is not unreasonable since absolute measurements of $T_{e}$ and $n$ were used to determine $\lambda_{D}$.

The theoretical curve in Fig. 8 is derived from the contour plots given in Section 3. Equation (8) from the convective saturation model is used to specify 
$k_{y 2}$ on the experimental plot in terms of $k_{\perp 2}$ taken from the theoretical contour plots. A plot of $\omega_{1} / \omega_{0}$ vs $k_{y 2} \lambda_{D}$ depends critically on the choice of $k_{\mid 2} \lambda_{D}$ as seen in Figs. 3 and 5. For comparison with experimental data, the value of $k_{\| 2} \lambda_{D}$ at which the growth rate is a maximum was used. The theoretical curves for co-directional and contra-directional sideband group velocities are nearly identical for this choice, and the experimental data of Fig. 8 bear this out.

Figure 9 shows experimental and theoretical values of $k_{\| 2} \lambda_{D}$ vs $\omega_{1} / \omega_{0}$. The difference between co-directional sidebands and contra-directional sidebands is very apparent, with co-directional waves having the shorter wavelength as expected from the constraint on $k_{\| 2}$ given by (4) and the condition $\omega_{1} / k_{\| 1,} \cong v_{t e} / \sqrt{2}$. As in Fig. 8 there is a systematic error factor in $k_{\mid 2} \lambda_{D}$, with theoretical values being about $\frac{2}{3}$ of the experimental values.

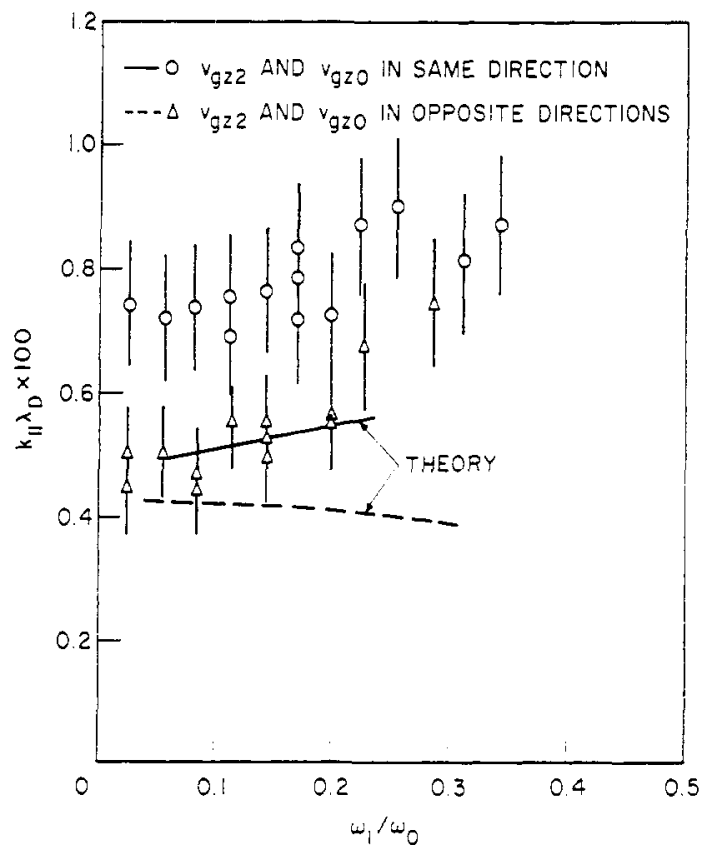

FyG. 9.-Measured sideband parallel wavenumbers $k_{2} \lambda_{D}$ is normalized frequency $\omega_{1} / \omega_{0}$ of the quasi-mode for the co-directional and contra-directional cases. The theory curves are taken at the point of maximum growth rate for a given $\omega_{1} / \omega_{0}$. Error bars represent relative error among the data points.

Measurement of $\lambda_{\mid 2}$ was complicated by the fact that the plasma was sensitive to the position of the endplate, which had to be moved axially to measure $\lambda_{\mid 2}$. Generally only $\frac{1}{2}$ to 1 wavelength was measured before the plasma conditions shifted.

The threshold for the quasi-mode decay process occurs when the imaginary part of the quasi-mode frequency exceeds the damping term of the sideband fluctuation. The damping is due to Landau damping and collisions. Threshold observations were made using the spectrum analyzer in the $\mathrm{CW}$ mode by observing the onset of decay spectra directly, or in the pulsed mode with the spectrum analyzer tuned to a decay-wave frequency and noting the power level at which amplification above noise first occurs. The observed threshold value for 
destabilization was somewhat fuzzy but generally occurred for input powers of $0.5-1.0 \mathrm{~W}$ (see Fig. 7). Based on the double-probe calibration referred to in Section 4 this corresponds to electric fields of $4-6 \mathrm{~V} \mathrm{~cm}^{-1}$. The calculations of Section 3 predict a threshold of $10 \mathrm{~V} \mathrm{~cm}^{-1}$, in fair agreement with the observation. Possible reasons for the difference are the inaccuracies in the electric field measurement (estimated at $\pm 50 \%$ ) and in the plasma parameters that were used for calculating the theoretical threshold.

In addition to spectrum and wavelength measurements, studies were made of convective saturation effects when the pump field was far above threshold. A pulsed pump wave was used because it allowed higher pump powers and minimized changes in the plasma density. The final amplification of both decay waves above the background noise was measured as a function of time using the spectrum analyzer as a tuned receiver. The probe was located where the amplitudes of the decay waves were maximum. Figure 10 is a plot of final amplification versus the pump power input to the power divider. As expected, the quasi-mode and sideband initially grow the same amount as a function of power. The small differences between the two curves are likely due to the plasma conditions drifting between the times the curves were taken, or possibly the difference in probe sensitivity at the two frequencies. The amplification is directly proportional to input power below $20 \mathrm{~W}$. Above $20 \mathrm{~W}$ the final amplitude increases more slowly with input power. However, as shown by the third curve in Fig. 10, this is also true of the detected pump-wave power. This suggests that at high powers the pump wave does not penetrate as well into the interior, perhaps

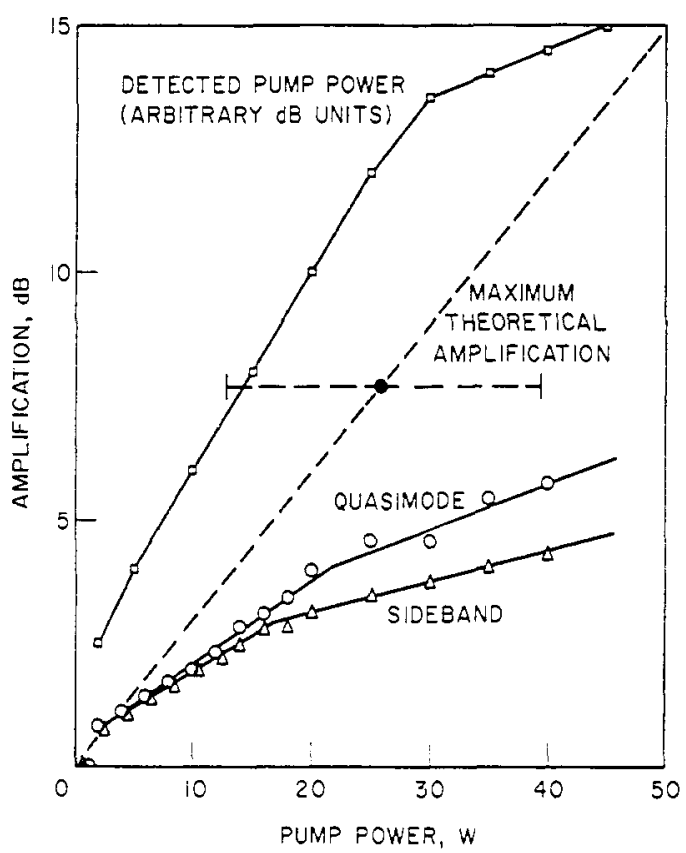

FiG. 10.-Final amplification (after reaching a steady-state in time) above background noise level of sideband and quasi-mode. The threshold pump power is approximately $0.7 \mathrm{~W}$. The amplification is proportional to pump power for levels far above threshold. An anomalous reduction in detected pump power per watt applied power occurs above $20 \mathrm{~W}$. 
due to reduced coupling caused by ponderomotive effects at the plasma surface as discussed by CHAN and CHIU (1979).

Based on the model of convective saturation given in Section 2, the maximum theoretical amplification of the decay waves vs pump power is shown by the dotted line in Fig. 10. The error bar on the theory curve is an estimate of the accuracy of the double-tipped probe electric field calibration which relates the pump electric field used in calculating the theory curve to pump power. Although Fig. 10 shows that about $10 \mathrm{~dB}$ amplification is expected for $40 \mathrm{~W}$ input power, it was observed to vary between 5 and $20 \mathrm{~dB}$ depending on the plasma conditions and probe position. The convective saturation model is consistent with the observed saturation levels, although other explanations are also not ruled out.

Radial probe scans of the decay waves showed decay wave amplitudes which are small on the inside edge (nearest plasma center) of the pump resonance cone and increase to a maximum on the outside edge of the pump resonance cone. This also agrees qualitatively with the theoretical expectation for a convecting decay wave propagating co-directionally. Contra-directional decay waves were probably disturbed by the probe mount because they propagated in the plasma region shadowed by the probe.

\section{SUMMARY AND CONCLUSIONS}

The parametric decay of a lower-hybrid wave into a lower-hybrid sideband and a non-resonant low-frequency quasi-mode was observed in a linear magnetized plasma device. The new contributions of this work are the measurement of the dispersion properties of this process inside the spatially localized region formed by the resonance cone of the lower hybrid pump wave, and the comparison of amplification with the convective saturation model. The decay process was modeled theoretically by the use of the uniform plasma parametric decay model for the region inside the pump-wave resonance cone and the assumption of zero growth rate elsewhere. The experimental results were in reasonable agreement with calculations based on this model.

The quasi-mode decay process was most clearly identified by the broad frequency spectrum of the decay waves. The quasi-mode frequency for maximum decay wave amplitude was generally $10-20 \%$ of the pump frequency. This frequency along with the parallel wavenumber measurements satisfied the approximate condition for non-linear Landau damping: $\omega_{1} / k_{11}=v_{\text {te }} / \sqrt{2}$. The decay-wave spectrum picked up by the probes was largely localized inside the pump-wave resonance cone.

The wavenumber vs frequency of the sideband decay wave was measured by a test-wave technique. The curve for sideband wavenumber $k_{y 2} \lambda_{D}$ ( $\hat{Y}$ in $\mathbf{E}_{0} \times \mathbf{B}_{0}$ direction) versus normalized quasi-mode frequency $\omega_{1} / \omega_{0}$ agreed with theory except for a numerical factor of $\frac{2}{3}$. Much of this discrepancy is probably due to a systematic inaccuracy in the Debye length $\lambda_{D}$. which was calculated from measured density and temperature.

The value of $k_{i 2} \lambda_{D}$ which was chosen for comparison with theory was that which corresponded to the maximum decay wave amplification. This method is not entirely satisfactory because the maximum is very broad and it is not clear why other values of $k_{12}$ could not also be present. The probes used to launch and 
detect the test wave may have some role in determining which $k_{\| 2}$ is detected. The agreement between theory and experiment was good considering the approximations and possible sources of error. The sideband parallel wavenumber was measured with the test-wave technique. A small difference between wavenumbers of co-directional sidebands and contra-directional sidebands was predicted and observed.

The experimental results are consistent with the model of convective saturation. The observed values of decay wave amplification above noise agree qualitatively with this model, but as shown in Fig. 10 there is a large uncertainty in the pump electric field and therefore quantitative agreement cannot be verified. The observed amplification increased linearly with pump power (at least initially) in agreement with the convective model. The wavelength measurements were done near threshold where $\varepsilon_{0} E_{0}{ }^{2} / n k T_{e} \simeq 0.001$, but the range of r.f. power used made this parameter as high as 0.01 , which exceeds the value expected near the central region of the plasma in current Tokamak heating experiments.

The energy content of the decay waves in this experiment was small compared to that of the pump, and no noticeable decrease in pump strength was caused by the instability. However, in Tokamaks the final amplification of the decay waves may be increased by wider pump widths, higher initial fluctuation levels, longer path lengths of wave propagation, and operation with $\omega_{0} / \omega_{\mathrm{LH}}$ smaller than the value of 2.5 used in the present experiment. The pump wave may then be gradually depleted as it penetrates. Equations (2) and (5) predict that $\Gamma$ will more than double if $\omega_{0} / \omega_{L H}$ changes from 2.5 to 1.5 , leading to stronger parametric decay. An opposing factor, however, would be the increasing Landau damping of the lower sideband as the electron temperature approaches $10 \mathrm{keV}$. Higher thresholds for the parametric instability would then result, possibly suppressing the quasi-mode parametric decay process in fusion plasmas.

\section{REFERENCES}

Bellai P. M. and Porkolab M. (1976) Physics Fluids 19, 995.

Bonizzoni G.. Fontanesi M., Grosso G., Lazzaro E. and Sndoni E. (1977) Plasma Physics 19, 1163.

BRowN I. G. and SHEPHERd V. (1967) Plasma Physics 9, 741.

CHAN V. S. and CHIU S. C. (1979) Physics Fluids 22, 1724.

Chavg R. P. H. and Porkolab M. (1974) Phys. Rev. Lett. 32, 1227.

Colestock P. L. and GetTy W. D. (1976) Physics Fluids 19, 1229.

Porkolab M. (1974) Physics Fluids 17, 1432.

PORKOLAB M. (1977) Physics Fluids 20, 2058.

PORKOLMB M. (1978) Nucl. Fusion 18, 367.

Porkolab M., Bermabi S., Hooke W. M., Motley R. W. and Nagashima T. (1977) Phys. Rev. Letr. 38, 230.

Stevens J. E. and GetTy W. D. (1980) IEEE Trans. Plasma Sci. PS-8, 18.

VILLAlon E. and Bers A. (1980) Nucl. Fusion 20, 243. 